\title{
Superluminal Hydrogen Atom in a Constant Magnetic Field in (3+1)-dimensional Spacetime (II)
}

\author{
Emmanuel D. K. Gazoya ${ }^{1^{*}}$ \\ ${ }^{1}$ Department of National Nuclear Research Institute (NNRI), Accelerator Research Center (ARC), \\ P.O. Box LG 80, Ghana Atomic Energy Commission (GAEC), Accra, Ghana.
}

Author's contribution

The sole author designed, analyzed and interpreted and prepared the manuscript.

Article Information

DOI: 10.9734/BJAST/2017/34195

Editor(s):

(1) Shahin Atashbar Tehrani, Particle Physics, Amirkabir University of Technology, Tehran, Iran.

Reviewers:

(1) A. Ayeshamariam, Khadir Mohideen College, Adirampattinam, India. (2) P. A. Murad, Morningstar Applied Physics, LLC, Vienna, Virginia. Complete Peer review History: http://www.sciencedomain.org/review-history/19510

\begin{abstract}
As has usually been the case in the tradition of some scientific novel ideas, we use the hydrogen atom as a "test particle", in the context of superluminal dynamical system theory. In Paper (I) of this series, the fundamental effect of an applied external magnetic field on a transversely guided beam of hydrogen-like atoms is uncovered, that of transformation from spherical wave expansion into plane wave function. This leads to an unprecedented concept of a planar helical hydrogen field, with a continuum of linear momentum in $(3+1)$-dimensional spacetime. Thereupon, we investigate a possible "superluminality" of this field. It turns out that, as in the case of a free Dirac field, the Dirac-hydrogen field accords with the law of conservation of energy and momentum. As a result, the generalized translational velocity component expectation value of the planar Dirac-hydrogen is found to exceed the speed of light, with however, a subluminal minimal velocity.
\end{abstract}

Keywords: Single-particle interpretation of the plane free Dirac wave; free Dirac particles.

\footnotetext{
*Corresponding author: E-mail: gazoya2001@yahoo.com;
} 


\section{INTRODUCTION}

The hydrogen atom has a long history of offering a testing ground for new physical theories, due to the simplicity of its unique bound state structure, consisting of a single electron and a much heavier proton and so, very easy to treat theoretically, and experimentally. The scientific research history on this atom started with the discovery of its spectral lines, produced by the transitions of the electron from higher excited states to the lower first excited one above the ground state, with corresponding wavelengths (also called Balmer series) given by [1]:

$$
\frac{1}{\lambda_{n}} \propto \frac{1}{2^{2}}-\frac{1}{n^{2}}, \quad n \in \mathbb{N}
$$

seconded by Rydberg in 1888 with his formula ,

$$
\frac{1}{\lambda_{f_{i}}}=\operatorname{Ryd}\left(\frac{1}{n_{i}^{2}}-\frac{1}{n_{f}^{2}}\right), \quad n_{f}, n_{i} \in \mathbb{N}, n_{f}>n_{i},
$$

This was later identified with the principal quantum number of the hydrogen atom, where the Rydberg constant Ryd amounts to approximately $1.1 \times 10^{7} \mathrm{~m}^{-1}$, corresponding to $13.6 \mathrm{eV}$. The series of spectral lines related to transitions to the ground state were found in the ultra violet [2] and more spectral series of the atom had been discovered in later years at lower energies. It was up until 1913 that the first theoretical explanation on the hydrogen spectrum was given by Bohr, in a series of papers. The model was that of an electron orbiting the proton on classical trajectories, like a planet orbits the sun; the angular momentum of the electron is set to be quantized, giving by $L=n \in \mathbb{N}$, thus inducing discrete energy levels:

$$
E_{n}=-\frac{R y d}{n^{2}}, \quad R y d=\frac{m \alpha^{2}}{2},
$$

where $m$ is the reduced mass of the electron in the hydrogen atom and $\alpha$ the fine-structure constant, introduced [3]. The transitions between the different Bohr levels which lead to the emission of photons with wavelengths expressed by formula (1.2), gave rise to the Rydberg constant.

Earlier, it was found that the spectral lines were actually multiplets of lines very close together [4].
At first this had been somehow explained in the more refined Bohr-Sommerfeld model [3], but it was only with the advent of the theory of quantum mechanics that a more satisfying answer could be obtained.

Reference [5] introduced a new perspective on the infinitesimal processes of atoms, which was elaborated rigorously later [6] [7]. This is presently known as the operator formalism. Using this new formalism [8] was able to derive the Bohr energy levels (1.3). [9] soon followed with the introduction of the wave function with its equation named after him. From this equation he derived the energy levels (1.3) and, with the wave function, determined the spatial distribution for the probability of finding the electron in the hydrogen atom. Soon later, [10] developed the quantum theory, based on the principles of special relativity. The Dirac equation incorporated the spin of the electron and reduces to the Schrödinger equation in the nonrelativistic limit. This equation can also be solved for the hydrogen atom [11] and features the line splittings [4], which are due to the electron spin, that is, spin-orbit coupling, and relativistic corrections.

Meanwhile, in 1922, Stern and Gerlach came up with an impressive experiment originally designed to test "space quantization" and consists of passing a beam of hydrogenlike (silver) atoms through a transverse inhomogeneous magnetic field. In addition to the result of space quantization, it was later realized, by the first proposal concerning the spin of the electron [12], that this experiment is quintessential in illustrating the intrinsic spin angular momentum of the electron. But that was not the end of the story about the multifunctional aspect of this experiment. Presently, wave transformation from spherical propagation to planar expansion (in the special case of guided hydrogen-like beams through a transverse magnetic field), is another fundamental and interesting aspect uncovered in Paper (I) of this series.

It is our aim in this second paper to draw the essential scientific implications of this transformation. As a result, we want to study the planar helical Dirac-hydrogen field, comparatively 
to the free Dirac plane wave, and derive the expectation value of the generalized linear velocity component thereof. In Section 2 , we review, summarily, the theory of the effective Lagrangian of the electromagnetic field. In Section 3, we study the coupled Maxwell and Dirac-hydrogen field, its solution in the limit of a homogeneous magnetic field, and evaluate its translational velocity component.

\section{THE EFFECTIVE LAGRAN- GIAN OF THE ELECTRO- MAGNETIC FIELD}

The Lagrange density of the massless spin-1 field, which produces the following inhomogeneous Maxwell field equation of second order

$$
\square A^{\mu}-\partial^{\mu}\left(\partial_{\nu} A^{\nu}\right)=j^{\mu}
$$

agrees with the Lagrangian

$$
\mathcal{L}_{0}=-\frac{1}{4} F_{\mu \nu} F^{\mu \nu}-j_{\mu} A^{\mu},
$$

up to a four-divergence term

$\partial_{\mu}\left(A^{\mu} \partial_{\nu} A^{\nu}-A^{\nu} \partial_{\mu} A^{\mu}\right)$, where $A^{\mu}$ is the fourpotential, $j^{\mu}$ the four-current, and $F_{\mu \nu}$ and $F^{\mu \nu}$ are defined below.

If we consider the electromagnetic field in isolation, it satisfies the linear Maxwell equations, and the superposition principle [i.e., solutions can be linearly superposed and the relation between wave functions at different times $(\psi(x, t)$ and $\left.\psi\left(x, t_{0}\right)\right)$ has to be linear], holds. There are no charge in empty space in the classical theory, and since the photons do not bear charge, and thus do not interact among themselves, their field is described by the free non-interacting Lagrange density [13].

To start with we recall that there are two Lorentz-invariant quantities that characterize the electromagnetic field, namely

$$
I_{1}=\boldsymbol{E}^{2}-\boldsymbol{H}^{2}, \quad I_{2}=\boldsymbol{H} \cdot \boldsymbol{E} .
$$

where $\boldsymbol{E}$ and $\boldsymbol{H}$ denote the electric and magnetic field strengths.

The vacuum of quantum electrodynamics (QED) is a polarizable medium owing to virtual processes and obtains novel physical properties. One may try to describe this effect by replacing the Lagrangian $\mathcal{L}_{0}$ of the electromagnetic field by an effective Lagrangian $\mathcal{L}_{\text {eff }}$. This will contain corrections in higher orders in $\boldsymbol{E}$ and $\boldsymbol{H}$ and lead to nonlinear field equations. In the limiting case of a stationary and homogeneous electromagnetic field an "exact" closed expression can be given for $\mathcal{L}_{\text {eff }}$. This result was found in a pioneering work [14]. Following in part another derivation [15], one can start by expressing the effective Lagrangian as a function of the invariants $I_{1}$ and $I_{2}$ :

$$
\begin{aligned}
\mathcal{L}_{e f f}(\boldsymbol{H}, \boldsymbol{E}) & =\mathcal{L}_{\text {eff }}\left(I_{1}, I_{2}\right) \\
& =\mathcal{L}_{\text {eff }}\left(\boldsymbol{H}^{2}-\boldsymbol{E}^{2}, \boldsymbol{H} \cdot \boldsymbol{E}\right) .
\end{aligned}
$$

The scalars $I_{1}$ and $I_{2}$ can be obtained by contraction of the electromagnetic field tensor $F^{\mu \nu}$, which is defined by

$$
\begin{aligned}
F^{\mu \nu} & =\partial^{\mu} F^{\nu}-\partial^{\nu} F^{\mu} \\
& =\left(\begin{array}{cccc}
0 & -E_{1} & -E_{2} & -E_{3} \\
E_{1} & 0 & -H_{3} & H_{2} \\
E_{2} & H_{3} & 0 & -H_{1} \\
E_{3} & -H_{2} & H_{1} & 0
\end{array}\right)(2.5)
\end{aligned}
$$

We also introduce the dual-field obtained by contracting $F_{\mu \nu}$ with the completely antisymmetric unit tensor (the Levi-Civita tensor)

$$
\begin{aligned}
{ }^{\star} F^{\mu \nu}= & \frac{1}{2} \varepsilon^{\mu \nu \lambda \sigma} F_{\lambda \sigma}=F^{\mu \nu}(\boldsymbol{E} \leftrightarrow \boldsymbol{H}) \\
= & \left(\begin{array}{cccc}
0 & -H_{1} & -H_{2} & -H_{3} \\
H_{1} & 0 & -E_{3} & E_{2} \\
H_{2} & E_{3} & 0 & -E_{1} \\
H_{3} & -E_{2} & E_{1} & 0
\end{array}\right)
\end{aligned}
$$

We may construct two scalars by contraction of these tensors, namely

$$
\begin{gathered}
F^{\mu \nu} F_{\mu \nu}=2\left(\boldsymbol{H}^{2}-\boldsymbol{E}^{2}\right)=2 I_{1}, \\
F^{\mu \nu \star} F_{\mu \nu}=-4 \boldsymbol{H} \cdot \boldsymbol{E}=-4 I_{2} .
\end{gathered}
$$

The Lagrange function is gauge invariant since it depends only on the field strengths. We will calculate the energy $W_{0}$ of the vacuum per unit volume as a function of the field strength. We sum up the energy eigenvalues $\epsilon_{p \sigma}<-m$ of all the electrons in the "Dirac sea" to obtain the total energy $E_{0}$. From this value the potential energy $U_{0}$ in the electric field has to be subtracted. The energy $E_{0}$ contains the potential energy $U_{0}$ of the 
electrons of the Dirac sea in the external field in addition to the pure energy $W_{0}$ of the vacuum. Since we are interested only in the pure energy of the vacuum, the contribution $U_{0}$ has to be subtracted from $E_{0}$ :

$$
\begin{aligned}
& W_{0}=E_{0}-U_{0}, \quad E_{0}=\sum_{p \sigma} \varepsilon_{p \sigma}^{(-)}, \\
& U_{0}=\sum_{p \sigma} \int d^{3} x \psi_{p \sigma}^{(-) \dagger} e A_{0}(x) \psi_{p \sigma}^{(-)},
\end{aligned}
$$

where $A_{0}(x)$ is the electrostatic potential. Here the sum extends over all momenta $p$ and all spin directions; only the states with negative energy $(-)$ are taking into account. $U_{0}$ may be expressed in terms of $E_{0}$ through the following general considerations valid in quantum mechanics.

Let $\hat{H}(\lambda)$ be a self-adjoint Hamiltonian that depends analytically on a parameter $\lambda$ and $\psi_{n}(\lambda)$ a normalized eigenfunction

$$
\hat{H}(\lambda) \psi_{n}(\lambda)=\varepsilon_{n}(\lambda) \psi_{n}(\lambda) .
$$

The derivative of the energy eigenvalue with respect to $\lambda$ then obeys, by differentiation of (2.11) and projection unto $\left\langle\psi_{n}\right|$

$\frac{\partial \varepsilon_{n}}{\partial \lambda}=\left\langle\psi_{n}\left|\frac{\partial \hat{H}}{\partial \lambda}\right| \psi_{n}\right\rangle+\left\langle\left|\left(\hat{H}-\varepsilon_{n}\right) \frac{\partial}{\partial \lambda}\right| \psi_{n}\right\rangle$.

The last term is zero since $\left\langle\psi_{n}\right| \hat{H}=\left\langle\psi_{n}\right| \varepsilon_{n}$.

Using this general statement by writing for the potential of a stationary, homogeneous $E$ field

$$
A_{0}(x)=-\boldsymbol{E} \cdot \boldsymbol{x}
$$

and considering the parameter $\lambda$ as the field strength, we have

$$
U_{0}=\boldsymbol{E} \cdot \sum_{p \sigma} \int d^{3} x \psi_{p \sigma}^{(-) \dagger} \frac{\partial \hat{H}}{\partial \boldsymbol{E}} \psi_{p \sigma}^{(-)}=\boldsymbol{E} \cdot \frac{\partial E_{0}}{\partial \boldsymbol{E}}
$$

and hence

$$
W_{0}=E_{0}-U_{0}=E_{0}-\boldsymbol{E} \cdot \frac{\partial E_{0}}{\partial \boldsymbol{E}} .
$$

This relation serves to switch from the energy to the Lagrange function. The relationship between the energy (Hamiltonian) and the Lagrangian for a system having the generalized coordinates $q_{i}$ in general reads

$$
W=\sum_{i} \dot{q}_{i} \frac{\partial \mathcal{L}}{\partial \dot{q}_{i}}-\mathcal{L} .
$$

In electrodynamics the potentials $A_{0}$ and $\boldsymbol{A}$ play the role of the generalized coordinates $q_{i}$. Because of the relation $\boldsymbol{E}=-\dot{\boldsymbol{A}}-\nabla A_{0}$ and $\boldsymbol{H}=\nabla \times \boldsymbol{A}$, there is a dependence on a generalized velocity $\left(\dot{q}_{i}\right)$ in the Lagrangian only in the time derivative of the vector potential. But differentiation with respect to $\dot{A}$ is equivalent to differentiation with respect to $\boldsymbol{E}$. Hence (2.15) can also be written as

$$
W=\boldsymbol{E} \cdot \frac{\partial \mathcal{L}}{\partial \boldsymbol{E}}-\mathcal{L} .
$$

Thus we find that the charge of the Lagrangian density of the electromagnetic field is given, up to a sign, by the additional energy density $E_{0}$ :

$\mathcal{L}_{\text {eff }}=\mathcal{L}_{0}+\mathcal{L}^{\prime}, \quad$ with $\quad \mathcal{L}^{\prime}=-E_{0}^{(\text {renormalized })}$.

In the second equation of (2.15), we have indicated that the expression of (2.9) still has to be renormalized. In particular the energy of the vacuum in the absence of the electromagnetic field has to be subtracted, because it cannot be observed.

In order to calculate $E_{0}$ we restrict ourselves for the beginning to the case of pure magnetic field, $\boldsymbol{E}=0$. The energy eigenvalues can be given exactly according to (3.29), below, where $n=$ $0,1,2, \ldots$ and $\sigma=-1$. The density of states per momentum interval is $|e| H / 2 \pi$, cf. (3.29). Hence, it is found that

$$
\begin{aligned}
\mathcal{L}^{\prime}(\boldsymbol{E}=0, \boldsymbol{H}) & =-E_{0} \\
& =\int_{-\infty}^{+\infty} \frac{d p_{z}}{2 \pi} \frac{|e| H}{2 \pi} \sum_{n \sigma} \sqrt{m^{2}+p_{z}^{2}+|e| H(2 n+1+\sigma)} \\
& =\frac{|e| H}{(2 \pi)^{2}} \int_{-\infty}^{+\infty} d p_{z}\left(\sqrt{m^{2}+p_{z}^{2}}+2 \sum_{n=1}^{\infty} \sqrt{m^{2}+p_{z}^{2}+2|e| H}\right) .
\end{aligned}
$$


Here we have taken account of the fact that all states are doubly degenerate except for the levels with $n=0, \sigma=-1$. The states with quantum number $n, \sigma=+1$ and with $n-1, \sigma=-1$ have the same energy. Only for the state $n=0, \sigma=-1$ can such a partner not be found.

Obviously (2.13) is highly divergent. But one can split off a physically meaningful finite expression. Differentiating twice with respect to the parameter $\mathrm{m}^{2}$ and summing up the series using the integral representation

$$
\frac{1}{m^{2}}=\int_{0}^{\infty} d \eta e^{-m^{2} \eta}
$$

it can be shown that the effective Lagrangian of the electromagnetic field is obtained as

$$
\mathcal{L}^{\prime}(\boldsymbol{E}=0, \boldsymbol{H})=\frac{m^{4}}{8 \pi^{2}} \int_{0}^{\infty} d \eta \frac{e^{-\eta}}{\eta^{3}}\left(-\tilde{H} \eta \operatorname{coth}(\tilde{H} \eta)+1+\frac{1}{3}(\tilde{H} \eta)^{2}\right),
$$

with $\tilde{H}=H / H_{c r}=|e| H / m^{2}$.

The investigation is more difficult for general electromagnetic fields, because an expression analogous to (3.29) cannot be found. However, the case of a constant pure electric field $(\boldsymbol{H}=0)$ can be reduced to (2.20) [13]. On another hand, in a uniform, pure magnetic field $(\boldsymbol{E}=0)$ with vector potential $\boldsymbol{A}=(0, H x, 0)$, the Lagrangian is invariant under translations in the $y$-direction. This last limiting case will be our testing ground for the generalized linear velocity component of the Dirac-hydrogen field.

\section{SUPERLUMINAL HYDRO- GEN ATOM IN (3+1)- DIMENSIONAL SPACETIME}

The transformation of spherical waves into plane waves is topically relevant, and has been presented earlier in the first article of this series. A hydrogen-like atom subject to an external magnetic field has its spherical wave expansion stabilized (or transformed) into helical plane wave propagation. The spherical-wave-formalism does no more apply in the study of hydrogen wave functions which propagate under the effect of applied magnetic field, since, the radius $r$ relative to the central potential becomes a constant of motion. We are thus led to introduce a new concept of hydrogen helical plane waves.

Consider the hydrogen atom with a single electron orbiting the dense and fixed nucleus with spin, in a radius $r$ (i.e., spin-orbit coupling), in the context of relativistic quantum theory using the Dirac equation. We restrict ourselves to realistic one-particle systems with positive energy, and waves propagating in the $z$-direction [16]. In this limit, with no essential lost of generality, the hydrogen atom is considered as one-body problem. The mass $m$ used in this context is the particle reduced mass $m=m_{e} /\left(1+m_{e} / m_{p}\right) \approx$ $m_{e}(1-1 / 1836)$ in an external Coulomb potential $V=-e^{2} / r$, derived from the attraction force of $e^{2} / r^{2}$ between electrons and protons of masses $m_{e}$ and $m_{p}$, respectively. The $z$-axis of our coordinate system is assumed to be parallel to the axis of the hydrogen atom.

\subsection{Coupled Maxwell and Dirac- hydrogen Fields}

The interaction of a Dirac-hydrogen with an electromagnetic field may be incorporated by the standard prescription from nonrelativistic quantum mechanics, resulting in a bound state with minimal coupling. We therefore insert the binding energy term $V=-e^{2} / r$ into the "Dirac-electron" equation subject to magnetic field. Quantum electrodynamics can describe this coupled system of Maxwell field and an electrically charged Dirac-hydrogen field, just as it does in the lesser complex unbounded case (see e.g., [17], Section 6.3) of Maxwell-Diracelectron field. This coupling culminates into the Dirac-hydrogen helical plane wave field, which is driven by the Dirac current $j^{\mu}=e \bar{\psi} \gamma^{\mu} \psi$. We will derive the classical equation of motion, and show that the energy-momentum vector of the total system is gauge invariant and that energy and momentum are conserved.

The Lagrangian of the system under consideration is given by 


$$
\begin{aligned}
\mathcal{L} & =\mathcal{L}_{\text {Dirac-hydrogen }}+\mathcal{L}_{\text {e.m. }}+\mathcal{L}_{\text {int }} \\
& =\bar{\psi}\left(i \gamma^{\mu} \partial_{\mu}-\frac{e^{2}}{r}-m\right) \psi-\frac{1}{4} F_{\mu \nu} F^{\mu \nu}-e \bar{\psi} \gamma^{\mu} \psi A_{\mu} .
\end{aligned}
$$

This leads to the following set of coupled equations of motion for the fields $\psi, \bar{\psi}$, and $A^{\mu}$ :

$$
\begin{aligned}
{\left[\gamma^{\mu} i\left(\partial_{\mu}+i e A_{\mu}\right)-\frac{e^{2}}{r}-m\right] \psi } & =0, \\
\bar{\psi}\left[\gamma^{\mu} i\left(\overleftarrow{\partial_{\mu}}-i e A_{\mu}\right)+\frac{e^{2}}{r}+m\right] & =0, \\
\square A^{\mu}-\partial^{\mu}(\partial \cdot A) & =e \bar{\psi} \gamma^{\mu} \psi,
\end{aligned}
$$

where the arrow in (3.3) indicates that the partial derivative acts on the function to the left. The Lagrangian (3.1) and the field equations (3.2), (3.3), and (3.4) are invariant under local gauge transformations

$$
\begin{aligned}
A_{\mu}^{\prime}(x) & =A_{\mu}(x)+\partial_{\mu} \Lambda(x), \\
\psi^{\prime}(x) & =\exp [-i e \Lambda(x)] \psi(x), \quad \bar{\psi}^{\prime}(x)=\exp [+i e \Lambda(x)] \bar{\psi}(x),
\end{aligned}
$$

where $\Lambda$ is an arbitrary scalar function. This is made possible by the prescription of minimal coupling, replacing the partial derivative by the "gauge-covariant" derivative, $\partial_{\mu} \rightarrow D_{\mu}=\partial_{\mu}+i e A_{\mu}$, and not forgetting that the additional term $-e^{2} / r$ describing the central hydrogen Coulomb potential is just as constant as the electron mass $m$ and its distance $r$ from the nucleus.

The canonical energy-momentum tensor resulting from (3.1) is

$$
\Theta^{\mu \nu}=\Theta_{\text {Dirac-hydrogen }}^{\mu \nu}+\Theta_{e . m .}^{\mu \nu}+\Theta_{i n t}^{\mu \nu},
$$

where

$$
\begin{aligned}
\Theta_{\text {Dirac-hydrogen }}^{\mu \nu} & =\bar{\psi} i \gamma^{\mu} \partial^{\nu} \psi-g^{\mu \nu} \bar{\psi}\left(i \gamma^{\sigma} \partial_{\sigma}-\frac{e^{2}}{r}-m\right) \psi \\
\Theta_{e . m .}^{\mu \nu} & =-F^{\mu \sigma} \partial^{\nu} A_{\sigma}+g^{\mu \nu} \frac{1}{4} F_{\sigma \tau} F^{\sigma \tau} \\
\Theta_{i n t}^{\mu \nu} & =g^{\mu \nu} e \bar{\psi} \gamma^{\sigma} \psi A_{\sigma} .
\end{aligned}
$$

Application of the gauge transformation (3.5) and (3.6) leads to the following extra terms

$$
\begin{aligned}
\triangle \Theta_{\text {Dirac-hydrogen }}^{\mu \nu} & =e \bar{\psi} \gamma^{\mu} \psi \partial^{\nu} \Lambda-g^{\mu \nu} e \bar{\psi} \gamma^{\sigma} \psi \partial_{\sigma} \Lambda, \\
\triangle \Theta_{e . m .}^{\mu \nu} & =-F^{\mu \sigma} \partial^{\nu} \partial_{\sigma} \Lambda, \\
\triangle \Theta_{i n t}^{\mu \nu} & =g^{\mu \sigma} e \bar{\psi} \gamma^{\sigma} \psi \partial_{\sigma} \Lambda .
\end{aligned}
$$

The sum of this contributions reduces to

$$
\begin{aligned}
\triangle \Theta^{\mu \nu} & =e \bar{\psi} \gamma^{\mu} \psi \partial^{\nu} \Lambda-F^{\mu \sigma} \partial^{\nu} \partial_{\sigma} \Lambda \\
& =\left(e \bar{\psi} \gamma^{\mu} \psi+\partial_{\sigma} F^{\mu \sigma}\right) \partial^{\nu} \Lambda-\partial_{\sigma}\left(F^{\mu \sigma} \partial^{\nu} \Lambda\right) \\
& =-\partial_{\sigma}\left(F^{\mu \sigma} \partial^{\nu} \Lambda\right)
\end{aligned}
$$

where in the last step the field equation (3.4) has been used. The energy-momentum four-vector proves to be gauge invariant since its change collapses to a surface integral:

$$
\begin{aligned}
\triangle P^{\nu} & =\int d^{3} x \triangle \Theta^{0 \nu}=-\int d^{3} x \partial_{\sigma}\left(F^{0 \sigma} \partial^{\nu} \Lambda\right)=\int d^{3} x \partial_{i}\left(E^{i} \partial^{\nu} \Lambda\right) \\
& =0
\end{aligned}
$$


Using the field equations $(3.2),(3.3)$, and (3.4), we can write the four-divergences of the contributions to the electromagnetic field-strength tensor as

$$
\begin{aligned}
\partial_{\mu} \Theta_{\text {Dirac-hydrogen }}^{\mu \nu} & =-\partial^{\nu}\left(\bar{\psi} \gamma^{\sigma} \psi\right) A_{\sigma} \\
\partial_{\mu} \Theta_{e . m .}^{\mu \nu} & =-\left(e \bar{\psi} \gamma^{\sigma} \psi\right)\left(\partial^{\nu} A_{\sigma}\right) \\
\partial_{\mu} \Theta_{i n t}^{\mu \nu} & =\partial^{\nu}\left(e \bar{\psi} \gamma^{\sigma} \psi\right) A_{\sigma}+\left(e \bar{\psi} \gamma^{\sigma} \psi\right)\left(\partial^{\nu} A_{\sigma}\right) .
\end{aligned}
$$

The conservation of energy-momentum in the combined systems follows from summing up the three contributions

$$
\partial_{\mu} \Theta_{\text {Dirac-hydrogen }}^{\mu \nu}+\partial_{\mu} \Theta_{e . m .}^{\mu \nu}+\partial_{\mu} \Theta_{i n t}^{\mu \nu}=0 .
$$

Clearly, this also holds for the symmetrized energy-momentum tensor of the symmetrized Lagrange density of the coupled Maxwell and Dirac-hydrogen fields.

Thus, the Dirac-hydrogen field, like the free Dirac spin-1/2 field, is in conformity with the basic natural law of conservation of energy and momentum, a quite significant result with the implication that these two fields share similar properties and can be quantized using the same treatment. In particular, in our restriction to constant magnetic field where variation of the action with respect to the field potential $A_{\mu}$ is practically null, and since the binding energy $-e^{2} / r$ and the field additional energy $|e| H(2 n+1+\sigma)$ terms remain constants of motion, one can check and find that the results obtained from second quantization of the Dirac-hydrogen field coincide with those of the free Dirac spin-1/2 field. These common results include notably the mirage of causality violation, the removal of which is achieved by superluminal field velocity prescription [16]. This also means that the general expression of the generalized variation transformation (as derived in [16], Section 3 ) of the free spin$1 / 2$ field, is applicable to the planar Dirachydrogen field. The variations, for intrinsic spin, orbital, and generalized angular momenta are all linear along the $z$-axis and their corresponding transformations are velocity transformations [16]. We will now seek the solution of the Dirachydrogen plane wave equation and subsequently evaluate the velocity of the field.

\subsection{Solution of the Dirac- hydrogen Equation in a Homogeneous Field Magnetic}

Applying the bispinor $\psi=\left(\begin{array}{l}\phi \\ \chi\end{array}\right)$ formalism of the Dirac equation ( [18], Chap. 2, e. 7 g.) to our special case of Dirac hydrogenic planar helical field, we calculate the energy of the propagation in the $z$-direction subject to an homogeneous magnetic field $\boldsymbol{H}=\mathrm{He}_{z}$ and reduce the problem to the differential equation of the harmonic oscillator, by elimination of $\chi$. Moreover, we evaluate the density of states per momentum interval in the volume $V=1$.

In general, states with definite momentum $\boldsymbol{p}$ are found with the ansatz

$$
\left(\begin{array}{c}
\phi \\
\chi
\end{array}\right)=\left(\begin{array}{c}
\phi_{0} \\
\chi_{0}
\end{array}\right) \exp \left[\left(\frac{i}{\hbar}\right) \boldsymbol{p} \cdot \boldsymbol{x}\right] \text {. }
$$

Equations (3.22) below are transformed into the same equations for components $\phi_{0}$ and $\chi_{0}$, but replacing the operator $(\hat{\boldsymbol{p}}-e \boldsymbol{A})$ by the eigenvalues $\boldsymbol{p}$.

The Dirac-hydrogen equation in two-component solution reads (in relativistic units $\hbar=c=1$ )

$$
\begin{aligned}
& i \frac{\partial}{\partial t} \phi=\boldsymbol{\sigma} \cdot(\hat{\boldsymbol{p}}-e \boldsymbol{A}) \chi+e A_{0} \phi-\frac{e^{2}}{r} \phi+m \phi \\
& i \frac{\partial}{\partial t} \chi=\boldsymbol{\sigma} \cdot(\hat{\boldsymbol{p}}-e \boldsymbol{A}) \phi+e A_{0} \chi-\frac{e^{2}}{r} \chi-m \chi
\end{aligned}
$$

The stationary solution for a constant purely magnetic field ( $A_{0}=0, \boldsymbol{A}$ independent of time) are obtained from

$$
\begin{aligned}
& \left(\varepsilon+\frac{e^{2}}{r}-m\right) \phi=\boldsymbol{\sigma} \cdot(\hat{\boldsymbol{p}}-e \boldsymbol{A}) \chi, \\
& \left(\varepsilon+\frac{e^{2}}{r}+m\right) \chi=\boldsymbol{\sigma} \cdot(\hat{\boldsymbol{p}}-e \boldsymbol{A}) \phi(3 .
\end{aligned}
$$

where the quantity $\varepsilon=\partial / \partial t$ describes the time evolution of the stationary state $\psi(x)$. We multiply the first equation by $\left(\varepsilon+\frac{e^{2}}{r}+m\right)$ and eliminate $\chi$ : 


$$
\left[\left(\varepsilon+\frac{e^{2}}{r}\right)^{2}-m^{2}\right] \phi=\boldsymbol{\sigma} \cdot(\hat{\boldsymbol{p}}-e \boldsymbol{A}) \boldsymbol{\sigma} \cdot(\hat{\boldsymbol{p}}-e \boldsymbol{A}) \phi .
$$

Next, we use the identity

$$
(\boldsymbol{\sigma} \cdot \boldsymbol{a})(\boldsymbol{\sigma} \cdot \boldsymbol{b})=\boldsymbol{a} \cdot \boldsymbol{b}+i \boldsymbol{\sigma} \cdot \boldsymbol{a} \times \boldsymbol{b}
$$

and the gradient property of the momentum operator $\hat{\boldsymbol{p}}=-i \nabla$ :

$$
\begin{aligned}
{\left[\left(\varepsilon+\frac{e^{2}}{r}\right)^{2}-m^{2}\right] \phi } & =[(\hat{\boldsymbol{p}}-e \boldsymbol{A})+i \boldsymbol{\sigma} \cdot(\hat{\boldsymbol{p}}-e \boldsymbol{A}) \times(\hat{\boldsymbol{p}}-e \boldsymbol{A})] \phi \\
& =\left[(\hat{\boldsymbol{p}}-e \boldsymbol{A})^{2}-e \boldsymbol{\sigma} \cdot \boldsymbol{H}\right] \phi \\
& =\left[\hat{\boldsymbol{p}}-2 e \boldsymbol{A} \cdot \hat{\boldsymbol{p}}+e^{2} \boldsymbol{A}^{2}-e \boldsymbol{\sigma} \cdot \boldsymbol{H}\right] \phi \\
& =\left[\hat{\boldsymbol{p}}^{2}+e^{2} H^{2} x^{2}-e H\left(\sigma_{z}+2 x \hat{p}_{y}\right)\right] \phi .
\end{aligned}
$$

The vector potential was chosen to be $\boldsymbol{A}=(0, H x, 0)$ in the last transformation, and $\nabla \cdot \boldsymbol{A}=0$ and $\boldsymbol{H}=\nabla \times \boldsymbol{A}$ have been used. We notice that the right-hand side of (3.25) obviously commutes with the components of the momentum operator $\hat{p}_{y}$ and $\hat{p}_{z}$. Consequently the ansatz

$$
\phi_{\sigma}(x)=e^{i\left(p_{y} y+p_{z} z\right)} f(x) \chi_{\sigma}
$$

presents itself where $\chi_{\sigma}$ is the unit spinor. Insertion into (3.25) immediately yields

$$
\left[\left(\varepsilon+\frac{e^{2}}{r}\right)^{2}-m^{2}\right] f(x)=\left(-\frac{d^{2}}{d x^{2}}+p_{y}^{2}+p_{z}^{2}+e^{2} H^{2} x^{2}-2 e H x p_{y}-e H \sigma\right) f(x),
$$

which can be written as

$$
\left[-\frac{d^{2}}{d x^{2}}+e^{2} H^{2}\left(x-\frac{p_{y}}{e H}\right)^{2}\right] f(x)=\left[\left(\varepsilon+\frac{e^{2}}{r}\right)^{2}-m^{2}-p_{z}^{2}+e H \sigma\right] f(x) .
$$

This is just the Schrödinger equation of the harmonic oscillator in the variable $\xi=x-p_{y} / e H$. The "oscillator energy" is given by $\hbar \omega=2|e| H$. The eigenvalues thus are $\lambda_{n}=(1+1 / 2) \hbar \omega=$ $(2 n+1)|e| H$. Therefore

$$
\left(\varepsilon+\frac{e^{2}}{r}\right)^{2}-m^{2}-p_{z}^{2}+e H \sigma=(2 n+1)|e| H
$$

or

$$
\varepsilon_{p \sigma}=-\frac{e^{2}}{r} \pm \sqrt{m^{2}+p_{z}^{2}+|e| H(2 n+1+\sigma)},
$$

$p_{z}$ is the momentum in the $z$-direction and $\sigma= \pm 1$ is the projection of the spin. The two signs of the time-evolution factor $\varepsilon_{p \sigma}$ correspond to two types of solutions of the Dirac-hydrogen equation, which we call positive and negative solutions, respectively. Except for the neutrino, the energy $\varepsilon^{\prime}= \pm \sqrt{m^{2}+p_{z}^{2}+|e| H(2 n+1+\sigma)}$ is the relativistic generalization of the Landau levels of a Dirac particle (spin-1/2 particles) in a magnetic field [13]. Hence, (3.29) may be regarded as the relativistic generalization of the Landau levels of a hydrogen-like atom in a magnetic field. Obviously, for the singleparticle interpretation limit of the plane Dirachydrogen wave (describing a realistic system with positive energy) to be possible, the positive quantity $+\sqrt{m^{2}+p_{z}^{2}+|e| H(2 n+1+\sigma)}$ in this last equation is required to be greater than the binding energy $-e^{2} / r$.

In order to determine the density of states, we note that the energy levels (3.29) are infinitely 
degenerate since the momentum $p_{y}$ does not appear in the formula. It is quite interesting to observe that in the classical framework, our solution describes a helical motion of the single spin-orbiting electron in a radius $r$ which carries the nucleus with chosen but quantized momentum components in $y$ and $z$ direction, and of horizontal displacement of locus

$$
x=\frac{p_{y}}{e H},
$$

along the $y$-axis . This is a charged system driven by the Dirac current $j^{\mu}=e \bar{\psi} \gamma^{\mu} \psi$, as mentioned above.

If we put the particle into a box with dimensions $L_{x}, L_{y}, L_{z}$, the $y$ and $z$ motions are quantized by the boundary conditions and the number of states reads $(\hbar=1)$

$$
\triangle N=\frac{L_{y}}{2 \pi} \triangle p_{y} \frac{L_{z}}{2 \pi} \triangle p_{z} .
$$

Now $\triangle p_{y}=(e H) \triangle x_{0}$ holds because of (3.30), where $x_{0}$ is a fixed center. We sum over the allowed values $0<x_{0}<L_{x}$ and obtain

$$
\triangle N=\frac{L_{y}}{2 \pi}|e| H L_{x} \frac{L_{z}}{2 \pi} \triangle p_{z}=\frac{|e| H}{(2 \pi)^{2}} \triangle p_{z} V .
$$

Next, the stationary states of the Dirac-hydrogen equation are found with the ansatz

$$
\psi(x, t)=\psi(x) \exp [-(i / \hbar) \varepsilon t]
$$

We want to determine, with no essential loss of generality, the wave function of the Dirachydrogen field propagating in the $z$-direction with positive energy. From (3.22), for fixed $\varepsilon=\varepsilon_{p \sigma}+$

$$
\begin{aligned}
& \frac{e^{2}}{r},(c=1) \\
& \chi=\frac{\boldsymbol{\sigma} \cdot(\hat{\boldsymbol{p}}-e \boldsymbol{A})}{\varepsilon+m} \phi=\frac{\boldsymbol{\sigma} \cdot \hat{\boldsymbol{\Pi}}}{\varepsilon+m} \phi,
\end{aligned}
$$

where we have set $\hat{\boldsymbol{\Pi}}=(\hat{\boldsymbol{p}}-e \boldsymbol{A})$. Applying the same treatment used in finding the free Dirac plane wave function [18], we calculate and obtain the Dirac-hydrogen plane wave function in the upward $z$-direction, with positive energy, as, (dropping the relativistic units)

$$
\begin{aligned}
& \psi_{\text {hydro }}=N\left(\begin{array}{c}
\left(\begin{array}{c}
1 \\
0
\end{array}\right) \\
\frac{c \sigma_{z} p_{z}}{m c^{2}+E_{p}}\left(\begin{array}{l}
1 \\
0
\end{array}\right)
\end{array}\right) \\
& \exp \left[i\left(p_{z} z-E_{p} t\right) / \hbar\right],
\end{aligned}
$$

where $E_{p}=+\varepsilon_{p \sigma}=-\frac{e^{2}}{r}+$ $\sqrt{m^{2}+p_{z}^{2}+|e| H(2 n+1+\sigma)}$ is the positive energy of the system required in order for the single-particle aspect to hold [16], and the factor $N$ is a normalization constant obtained as

$$
N=\sqrt{\frac{m c^{2}+E_{p}}{2 E_{p}} .}
$$

Thus, the Dirac-hydrogen plane wave function is similar, in terms of its expression, to the plane free Dirac wave (i.e., the free spin-1/2 field). Precisely, these two fields can be said to be identical up to two quantities: a binding factor $-e^{2} / r$ and an additional constant energy term $|e| H(2 n+1+\sigma)$. Now it is obvious to wonder what will be the difference, as far as propagation linear velocity is concerned, between the Dirachydrogen field and the free Dirac field.

\subsection{Evaluation of Velocity of the Dirac-hydrogen Atom in a Constant Magnetic Field in Spacetime}

According to our discussion at the end of Section 3.1 above, the implications, methodology, and prescriptions for velocity evaluation, used in [16] for the free Dirac field, hold in the study of the plane Dirac-hydrogen field. Its generalized angular momentum transformation expression, which is the composition of spin and orbital transformations, is therefore given by [16]:

$$
\begin{aligned}
\delta M & =\left[\begin{array}{lll}
\left.M_{\delta S_{03}} \circ M_{\delta L_{03}}\right] \boldsymbol{x}_{\mu}=[\mathcal{F}(V)] \boldsymbol{x}_{\mu} \\
= \\
& \cos (V / c) & -\cos (V / c)
\end{array}\right)\left(\begin{array}{l}
t \\
x \\
y \\
z
\end{array}\right),
\end{aligned}
$$


where $V$ is the generalized (overall) relative translational velocity component of the system and $c$, the speed of light.

Here now comes to take shape the difference between the two fields. Though the two propagations are helical in spacetime, the atomic linear momentum is solely produced by spinorbit coupling of the single electron in the limit of a fixed definite radius $r$ about the nucleus, whereas the free electron dynamics helicity is carried out on an indefinite, unclear radius basis. Hence, the geometry of the helix described by the spin-orbiting electron in the fixed radius $r$ about the nucleus becomes a central ingredient in the velocity evaluation of the hydrogen field.

Some helpful relationships emerge from the geometry of the cylinder formed by one revolution of the helix. If the cylinder is split down the side, parallel to the $z$-axis, and laid flat, the wall of the cylinder forms a rectangle. The length of the sides parallel to the $z$-axis equals $\|\delta M\|$ (which is the length of the overall relative linear variation of the field in (3.36)). The length of the other sides equals the circumference of the cylinder $(2 \pi r)$, Fig. 1 (a) and (b). The arc of the helix for one revolution is the diagonal, with length equal to $\sqrt{\|\delta M\|^{2}+4 \pi^{2} r^{2}}=\left\|x_{\mu}\right\|$ (i.e., this arc of the helix or the diagonal is just the length of the fourvector $x_{\mu}$ which, stretched out, coincides with the angular momentum vector). Also crucial in these discussions is the angle $\theta$ formed by the diagonal and the side parallel to the $z$-direction. The diagonal is the arc of the helix, so $\theta$ is the angle formed by the $z$-axis and any line tangent to the helix, and is given by (see Fig. 1 (b) ):

$$
\tan \theta=\frac{2 \pi r}{\|\delta M\|}
$$

Thus, also, we have that

$$
\begin{aligned}
& \cos \theta=\frac{\|\delta M\|}{\sqrt{\|\delta M\|^{2}+4 \pi^{2} r^{2}}}=\frac{\|\delta M\|}{\left\|\boldsymbol{x}_{\mu}\right\|}=\frac{\|[\mathcal{F}(V)]\|\left\|\boldsymbol{x}_{\mu}\right\|}{\left\|\boldsymbol{x}_{\mu}\right\|} \\
& =\|[\mathcal{F}(V)]\|_{\infty}=\|[\mathcal{F}(V)]\|_{1}=\max _{1 \leq i \leq n} \sum_{j=1}^{n}\left|a_{i j}\right| \\
& \begin{array}{l}
=\left\|\left(\| \begin{array}{ccc} 
& & -\cos (V / c) \\
\cos (V / c) & \cos (V / c) &
\end{array}\right)\right\|_{\infty \text { or } 1} \\
=|\cos (V / c)|,
\end{array}
\end{aligned}
$$

i.e., simply put:

$$
\cos (V / c)=\cos \theta
$$

where the only nonzero entry $a_{i j}$ in each row and column of the matrix $[\mathcal{F}(V)]$ is equal to its eigenvalue $\cos (V / c)$, and the angle $\theta$ must be acute because of the absolute value notation.

So, this is to say that the angle $\theta$ between the $z$-axis and any line tangent to the helix (i.e., the diagonal formed by the arc of the helix) is just equal to the translational velocity ratio $\mathrm{V} / \mathrm{c}$ of the field motion. It is worthy of note that equation (3.38) or (3.39) corroborates and justifies, elegantly, the assumptions, claims, and prescriptions according to which the field velocity transformation matrix eigenvalue should be a trigonometric sine or cosine function with argument $V / c$, see [16], Section 3 . We will now determine the angle $\theta$, and this would readily result in the velocity $V$ evaluation of the Dirac-hydrogenic field. 


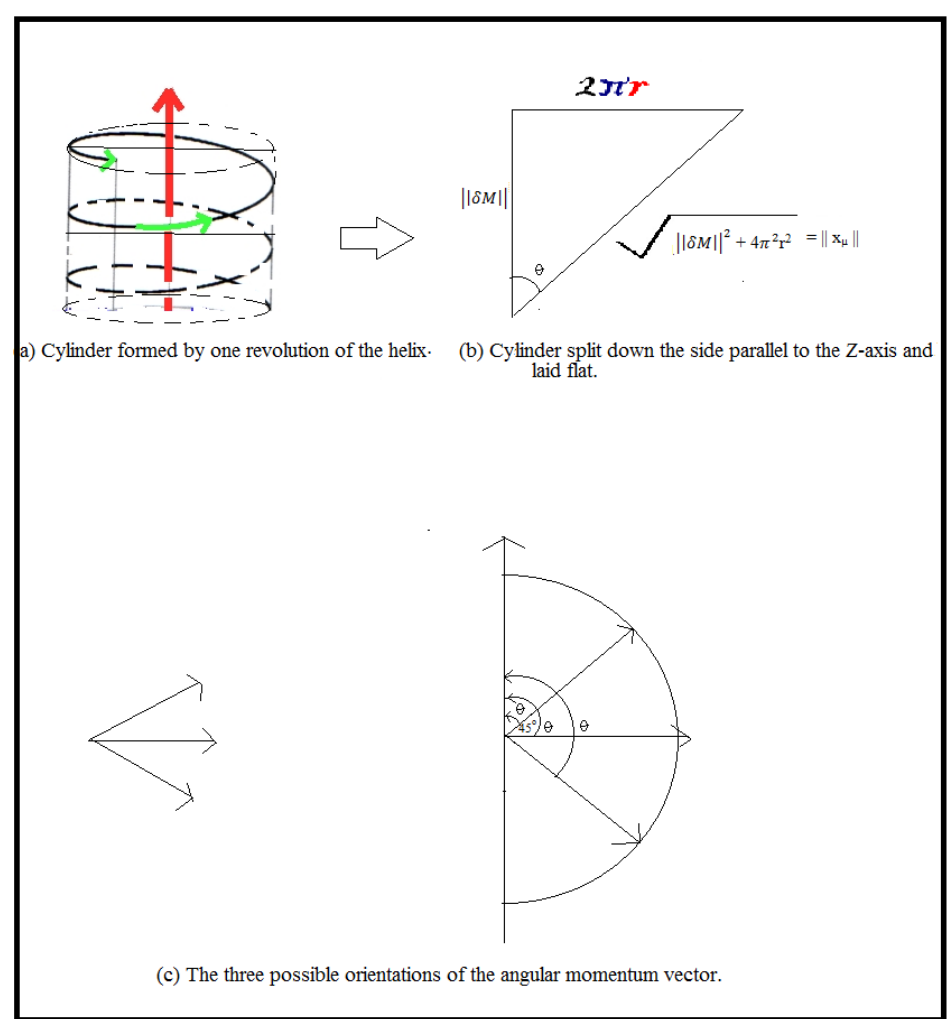

Fig. 1. Reduction of one revolution of hydrogen helical trajectory to the geometry of the cylinder.

The angle $\theta$ is a physical quantity which in a classical system may assume any value, but which in a quantum system may take on only certain discrete values. One needs not accept this result on faith. The magnetism exhibited by atoms (especially hydrogen-like ones) provides an experimental means by which one may study the direction of the angular momentum vector. There is a simple, elegant experiment which illustrates the quantization of $\theta$, just as a line spectrum illustrates the quantization of energy in the hydrogen atom [19]. It has been shown, by passing a narrow beam of atoms through a magnetic field placed perpendicular across the interior axis of an evacuated tube, that the angle $\theta$ between the angular momentum vector and the direction of the applied magnetic field (the $z$-axis) is found to equal only three values, $45^{\circ}$, $0^{\circ}$, and $-45^{\circ}$, [19], Fig. 1 (c).
Thereupon, subsequently readily, the hydrogenic field overall linear velocity component expectation value $V=V_{\text {supal } H}$ is obtained as:

1. for the case $\theta=45^{\circ}$, this corresponds to spin-up propulsion in the $z$-direction, and

$$
V_{\text {supal } H}=\left(\frac{\pi}{4}+2 k \pi\right) \times c, \quad k=0,1,2, \ldots
$$

2. for $\theta=0^{\circ}$, there is no linear momentum and so $V$ is identically zero, making this point trivial; and finally

3. the case $\theta=-45^{\circ}$ describes a spindown helical motion, with the same linear velocity of propagation as in spin-up.

Thus, as in the free Dirac field context, the Dirac-hydrogen field linear velocity component expectation value is of course quantized, and 
exceeds the speed of light with, however, a subluminal minimal value of

$$
V_{H-\text { minimal }} \simeq(0.7855) \times c .
$$

\section{DISCUSSIONS}

We observe that the subluminal minimal linear velocity component evaluated for the hydrogen atom, in contradistinction to the superluminal minimal velocity of a free Dirac particle in spacetime [16], has obviously to do with the binding energy and the constraint of quantization of direction of the angular momentum vector about the $z$-axis, exerted by the applied magnetic field.

As mentioned earlier in this work, it is important to note that spherical wave functions of hydrogenlike atoms cannot produce a continuum of linear angular momentum, because of the perturbation of the system caused by the unstable nuclear magnetic moment. That is the reason behind the fact that, to obtain a sustained linear momentum to carry these particles in spacetime, there must occur a transformation from spherical wave function into plane wave expansion, hence our interest in the Stern-Gerlach experiment, to this effect. It is admissible that this superluminal result for the hydrogen could hold for all other hydrogen-like atoms in this context of coupled Maxwell and Dirac fields (since, in general, the radius, i.e., the distance of the valence electron from the nucleus remains constant in motion), if their angular momentum vector exhibits experimentally similar direction quantization constraints. Furthermore, this result could imply another interesting one: the superluminal linear velocity of an electron in homogeneous magnetic field. How could it be otherwise, since, clearly, the Lagrangians of these two dynamical systems are identical, up to a binding constant energy term $V=-e^{2} / r$ ? Finally, it should be observed that though the free Dirac field and the Dirac-hydrogen field share common generalized angular momentum expression, their giro-factors (known to be 2 for the free electron, but yet to be determined for the bound state electron in hydrogen atomic orbital) are not the same. This triggers another future investigation to be achieved for the helical plane Dirac-hydrogen.

\section{CONCLUSION}

Gregory Breit, in 1928, demonstrated that a spin$1 / 2$ particle always moves with the speed of light. Walter Greiner, in 2000, went further to prove that this kind of particle always moves with the speed of light, either in free motion or when subject to a magnetic field. I have laboured to discern and explain beyond these results, in the present article and the previous ones as well on the same subject-matter, that free Dirac particles in general, an electron in a magnetic field, and an electron in bound state of hydrogen atomic orbital subject to a magnetic field, all of these could be superluminal. To paraphrase a great discoverer (I cannot mention here) who was desperate to be understood by readers, I have not compromised conscience to suit the general drift of thought (that regards any superluminal entity as paradoxical or "unnatural"), but have bluntly and honestly given the text of a "probable" scientific truth.

\section{COMPETING INTERESTS}

Author has declared that no competing interests exist.

\section{References}

[1] Balmer JJ. Notiz über die spectrallinien des wasserstos. Ann. Phys. 1885;261(80).

[2] Lyman T. The spectrum of hydrogen in the region of extremely short wave-lengths. Astrophys. 1906;J.23(181).

[3] Sommerfeld A. Zur Quantentheorie der Spektrallinien. Ann. Phys. 1916;356(1).

[4] Michelson AA. Application of interference methods to spectroscopic measurement. Publ. Astron. Soc. Pac. 1892;4(190).

[5] Heisenberg W. Über quantentheoretische Umdeutung kinematischer und mechanischer Beziehungen. Z. Phys. 1925;33(879).

[6] Born M, Jordan P. Zur Quantenmechanik. Z. Phys. 1925;34(858).

[7] Born M, Heisenberg W, Jordan P. Zur Quantenmechanik. 1925;34(1):858-888. 
[8] Pauli W. Über das wasserstospektrum vom standpunkt der neuen quantenmechanik. Z. Phys. 1926;36(336).

[9] Schrödinger E. Quantisierung als eigenwertproblem. Ann. Phys. 1926;384(361).

[10] Dirac PAM. The quantum theory of the electron. Proc. R. Soc. A. 1928;117(610).

[11] Gordon W. Die Energieniveaus des Wasserstoatoms nach der Diracschen Quantentheorie des Elektrons. Z. Phys. 1928;48(11).

[12] Uhlenbeck GE, Goudsmidt S. Naturw. 1925;13(953).

[13] Greiner W, Reinhardt J. Quantum electrodynamics. Second ed. SpringerVerlag, New-York, Berlin, Heidelberg. 1992;1994.

[14] Heisenberg W, Euler H. Z. Physik.1936;38(314).
[15] Weisskopf V. Kgl. Dankse Vid. Selskab. Math.-fys. Medd. 1036;XIV(166).

[16] Gazoya EDK, Oduro FT, Prempeh E. Investigation of superluminal motion of free spin-half particles in spacetime.

Available:

http://dx.doi.org/10.5539/

- jmr.v7n4p12, $201 \overline{5}$

[17] Greiner W, Reinhardt J. Field quantization. Second ed. Springer-Verlag, Berlin, Heidelberg, GmbH; 1996.

[18] Greiner W. Relativistic Quantum mechanics-wave equations. Third ed. Springer, Berlin, Heidelberg; 2000.

[19] Bader RFW. An introduction to the electronic structure of atoms and molecules. McMaster University, Hamilton, Ontario.

Available: http://www.chemistry.mcmaster. ca/esam/intro.html

(C) 2017 Gazoya; This is an Open Access article distributed under the terms of the Creative Commons Attribution License (http://creativecommons.org/licenses/by/4.0), which permits unrestricted use, distribution, and reproduction in any medium, provided the original work is properly cited. 